\title{
VIABILIDADE AGRONÔMICA DAS CULTURAS DE RÚCULA E DE ALMEIRÃO EM SISTEMA DE CULTIVO SOLTEIRO E CONSORCIADO
}

\author{
ANDREANI JUNIOR, Roberto ${ }^{1}$ \\ ROCHA, Alex Henrique da Silva ${ }^{2}$ \\ KOZUSNY-ANDREANI, Dora Inés ${ }^{3}$
}

\begin{abstract}
RESUMO: A eficiência de um sistema consorciado fundamenta-se principalmente na complementaridade entre as culturas envolvidas, sendo que esta será maior, a medida que se consegue minimizar os efeitos estabelecidos de uma espécie sobre a outra. Este sistema de cultivo se apresenta como um método prático e com vantagens ambientais, produtivas e econômicas. O objetivo do presente trabalho foi avaliar a viabilidade agronómica das culturas de rúcula e almeirão em sistemas de cultivo solteiro e consorciado . O delineamento experimental foi em blocos ao acaso com três tratamentos em seis repetições, sendo as culturas de rúcula e de almeirão em monocultivo e ambas as culturas em sistema consorciado. A colheita foi realizada aos quarenta e oito dias após a semeadura. Verificou-se que não houve diferença significativa para a massa fresca, a massa seca, o comprimento das raízes e a produtividade na cultura da rúcula em ambos os sistemas de cultivo. A cultura do almeirão apresentou diferenças significativas para a massa fresca e seca, comprimento das raízes e produtividade, com redução em todos os aspectos avaliados, porém apresentando padrão comercial. Considerando a produtividade, o IEA (índice de equivalência de área) atingiu 1,17, o que indicou a viabilidade do consórcio, otimizando práticas culturais como adubação, capinas e irrigação, além da geração de renda extra para o produtor.
\end{abstract}

Palavras-chave: Chicorium intybus L.. Eruca sativa Mill. Sistemas de produção. Produtividade.

SUMMARY: The efficiency of a consortium system is based mainly on the complementarity between the cultures involved, and this will be higher, as they will be able to minimize the effects established a species over another. This farming system is presented as a practical methods and environmental, productive and economic advantages. The aim of this study was to evaluate the agronomic viability of arugula and chicory cultures in monocropped and intercropped system. The experimental design was a randomized block with three treatments in six replications and cultures of arugula and chicory in monocropped and both crops in intercropping system. Plants were harvested forty eight days after sowing. There was no significant difference for fresh mass, dry mass, root length and productivity in the culture of the arugula in both cropping systems. The chicory cultivation showed significant differences for fresh and dry weight, root length and productivity, reduction in all aspects evaluated, but presenting commercial standard. Considering productivity, the IEA (land equivalent ratio) reached 1.17, which indicated the consortium's feasibility, optimizing cultural practices such as fertilizing, weeding and irrigation, as well as generating extra income for producers.

Palavras-chave: Chicorium intybus L. Eruca sativa Mill. Production systems. Productivity.

\section{INTRODUÇÃO}

As hortaliças apresentaram aumento de consumo nos últimos anos devido à preocupação e conscientização da população que busca uma alimentação mais diversificada e saudável. Para isso, é importante que os agricultores reduzam ou eliminem as deficiências do setor produtivo por meio da criação de sistemas que melhorem a produção de hortaliças (MONTEZANO ; PEIL, 2006). Uma técnica que tem sido empregada é através do sistema consorciado utilizada principalmente, por pequenos produtores, que assim, aproveitam ao máximo as áreas limitadas que dispõem. (CAETANO et al., (1999).

\footnotetext{
${ }^{1}$ Professor/Pesquisador da Unicastelo

${ }^{2}$ Engenheiro Agrônomo

${ }^{3}$ Professora/Pesquisadora da Unicastelo
} 
De acordo com Cecílio Filho e May (2002), entre as vantagens do cultivo consorciado em relação ao monocultivo, se destacam o aumento da produtividade por unidade de área; a possibilidade de produção diversificada de alimentos em uma mesma área, propiciando melhor distribuição temporal de renda; uso mais efetivo da mão-de-obra, aproveitamento mais adequado dos recursos disponíveis; aumento da produção vegetativa do solo contra a erosão; melhor controle de plantas invasoras que no cultivo solteiro, por apresentar alta densidade de plantas por unidade de área, gerando uma cobertura vegetal mais rápida do solo, além do sombreamento. (TEIXEIRA FILHO et al., 2005).

Para Heredia Zarate et al. (2003), caso ocorra de uma cultura ter problemas de ordem climática ou ataque de parasitas e ter sua produtividade reduzida, a outra cultura componente do sistema poderá compensá-la.

Apesar de apresentar várias vantagens ambientais, sociais e econômicas, o consórcio de hortaliças, apesar de muito praticado, ainda é pouco estudado (MONTEZANO ; PEIL, 2006). Uma das principais dificuldades encontradas é quanto as cultivares disponíveis no mercado. Negreiros et al., (2002), concluem que cultivares adaptadas ao sistema consorciado obteriam produtividade superior às utilizadas atualmente, oriundas para o monocultivo e ainda citam que as cultivares foram selecionadas para o monocultivo, não sendo possível assim prever o comportamento de genótipos em consórcios.

Objetivou-se com este trabalho avaliar a viabilidade agronómica das culturas de rúcula e almeirão em sistemas de cultivo solteiro e consorciado.

\section{MATERIAL E MÉTODO}

O experimento foi conduzido na área experimental da Fazenda de Ensino e Pesquisa da Universidade Camilo Castelo Branco/UNICASTELO, Campus Fernandópolis - SP, no período de junho a setembro de 2013. A cultivar utilizada para a cultura da rúcula foi "Apreciatta Folha Larga" que apresenta alto vigor de plantas, proporcionando uma maior precocidade nas mudas como também na produção, tendo excelente aceitação de mercado (PORTO, 2008). Já para a cultura do almeirão foi utilizada a cultivar "Radiche Folha Larga".

O delineamento experimental utilizado foi o de blocos casualizados, com três tratamentos e seis repetições. As parcelas possuíam medidas de 1,40 m de largura por 2,20 m de comprimento. As parcelas de rúcula e de almeirão em monocultivo estavam compostas por dez linhas com 0,20 m entre linhas e as parcelas consorciadas possuíam 0,10 m entre linhas, à área total da parcela no arranjo 1:1 foi de 3,08 $\mathrm{m}^{2}$, formada por fileiras simples de rúcula alternadas com fileiras simples de almeirão. A rúcula foi cultivada no espaçamento de $0,20 \mathrm{~m}$ x $0,04 \mathrm{~m}$ e o almeirão no espaçamento de $0,20 \mathrm{~m} \times 0,05 \mathrm{~m}$ no monocultivo, e 0,10 x 0,4 m para a rúcula e 0,10 x $0,05 \mathrm{~m}$ no almeirão no sistema de cultivo consorciado.

O solo foi preparado dez dias antes da instalação das culturas, quando foi realizado o seu revolvimento, adicionando-se material orgânico, constituído por composto de cama de frango $\left(5,0 \mathrm{t} \mathrm{ha}^{-1}\right)$; bokashi 2,0 $\mathrm{tha}^{-1}$ ) e termofosfato $1,5 \mathrm{tha}^{-1}$ ) como descrito por Oliveira et al. (2010)

. A rúcula e o almeirão foram semeados em semeadura direta e simultânea. Em ambas as culturas foram realizadas raleios quando às plantas estavam entre 4 e $5 \mathrm{~cm}$ de altura para a rúcula e entre 9-10 $\mathrm{cm}$ para o almeirão, deixando-se as plantas mais vigorosas.

Foi realizada a adubação de plantio com $150 \mathrm{~g} \mathrm{~m}^{2^{-1}}$ com adubo químico NPK na formulação 4-148 e adubação de cobertura após 28 dias da semeadura com adubo químico a base de sulfato de amônia (N), aplicando-se $150 \mathrm{~g} \mathrm{~m}^{2-1}$.

Durante o experimento foram realizadas capinas manuais e irrigações diárias pelo sistema de 
microaspersão.

A colheita da rúcula e do almeirão ocorreu aos 49 dias após a semeadura. As características valiadas foram: massa fresca, massa seca, comprimento da raiz . A massa fresca foi determinada por uma amostragem de vinte plantas retiradas aleatoriamente das parcelas, sendo dez plantas de cada parcela, ou seja, dez do sistema consorciado e dez do monocultivo, utilizando uma balança de precisão para aferir o peso, estimando-se a média e expressando-a em gramas. A massa seca foi determinada pela mesma amostragem de vinte plantas, submetidas em estufa de circulação de ar, a uma temperatura de $65^{\circ} \mathrm{C}$, pelo período de 72 (setenta e duas) horas, utilizando uma balança de precisão para aferir o peso, estimando-se a média e expressando-a em gramas.

O comprimento da raiz foi determinado pela mesma amostragem de vinte plantas, medindo-se o comprimento das raízes, estimando-se a média e expressando-a em centímetros. A produtividade total foi calculada com os valores da massa fresca e expressa em $\mathrm{t} \mathrm{ha}^{-1}$.

O índice de equivalência de área (IEA) é obtido a partir da relação entre a produtividade na área cultivada em consórcio e aquela em monocultivo (VIEIRA, 1984), utilizando a fórmula:

$$
I E A=\frac{\text { prod.de rúcula em consorcio }}{\text { prod.de rúcula em monocultivo }}+\frac{\text { prod.de almeirâo em consorcio }}{\text { prod.de almeirẫoem monocultivo }}
$$

Uma análise de variância para experimento fatorial em blocos completos casualizados foi realizada para avaliar as características de cada cultura. O teste de Tukey ao nível de 5\% de probabilidade foi usado na comparação das médias. O software utilizado na análise foi o ASSISTAT (ASSISTAT, 2012).

\section{RESULTADO E DISCUSSÃO}

Para a massa fresca (planta ${ }^{-1}$ ), massa seca e comprimento da raiz (Tabela 1), assim como para a produtividade (Tabela 2), verificou-se para a cultura da rúcula que não houve diferença significativa entre os sistemas avaliados, ou seja, comparando-se a mesma cultura no monocultivo com o cultivo consorciado. Porém em todas as características no sistema de consórcio, obteve-se uma tendência de redução se comparado à monocultura, porém não houve perda quanto ao padrão de qualidade comercial. De acordo com Gliesman (1990) citado por Oliveira et al. (2005), geralmente se observa uma redução na produtividade das espécies consorciadas e que essa redução no sistema de cultivo consorciado também pode ser associada a uma maior competição interespecífica onde se espera uma interferência maior de uma, no ambiente da outra, devido a maior proximidade das plantas componentes do consórcio (CECÍLIO FILHO, 2005).

Constatou-se que havendo uma tendência de queda na produtividade da rúcula (Tabela 2), a cultura apresentou um bom rendimento mesmo com um plantio adensado. Para a cultura do almeirão, a massa fresca $\left(\right.$ planta $\left.^{-1}\right)$ no sistema de monocultivo apresentou rendimento maior quando comparando com o almeirão em cultivo consorciado, com uma diferença mínima significativa de 10,92 (Tabela 1). Resultado similar foi obtido por Harder, Heredia Zarate e Vieira (2005), quando o almeirão cultivar "amarelo" foi consorciado com a rúcula cultivar "cultivada", atribuindo essa redução da massa fresca ao número de linhas por canteiro. Quanto à massa seca também se observou um maior rendimento no monocultivo quando comparado ao cultivo consorciado, com uma diferença mínina significativa de 1,00 (Tabela 1). No comprimento das raizes, observou-se que no monocultivo o desenvolvimento foi superior ao sistema de cultivo consorciado, com uma diferença mínina significativa de 1,45 (Tabela 1). 
$\mathrm{Na}$ produtividade, observou-se uma redução acentuada no sistema de cultivo consorciado quando se compara ao monocultivo, com uma diferença mínina significativa de 7,32 (Tabela 2). Vale ressaltar que mesmo com um decréscimo acentuado quanto à produtividade, a cultura do almeirão obteve em ambos os sistemas de cultivo, um padrão comercial. Um dos fatores que podemos atribuir a essa redução significativa no sistema de cultivo consorciado pode ser devido ao almeirão (Cichorium intybus L.) e a rúcula (Eruca sativa Mill.), apresentarem hábitos de crescimento semelhantes e períodos de maturação próximos (HARDER; HEREDIA ZARATE; VIEIRA 2005).

Tabela 1- Valores médios de rendimento de massa fresca e massa seca e comprimento do sistema radicular das culturas da rúcula e almeirão em monocultivo e cultivo consorciado.

\begin{tabular}{lccc}
\hline \multirow{2}{*}{ Modo de cultivo } & \multicolumn{3}{c}{ Produção } \\
\cline { 2 - 4 } & MF (g) & MS (g) & CR (cm) \\
\cline { 2 - 4 } & & Rúcula \\
\hline Monocultivo & $42,77 \mathrm{a}^{1}$ & $3,33 \mathrm{a}$ & $14,79 \mathrm{a}$ \\
Consorciado & $33,18 \mathrm{a}$ & $2,55 \mathrm{a}$ & $14,51 \mathrm{a}$ \\
CV\% & 52,71 & 54,01 & 19,82 \\
DMS & 12,84 & 1,02 & 1,86 \\
\hline & \multicolumn{3}{c}{ Almeirão } \\
\hline Monocultivo & $52,81^{\mathrm{a}}$ & $3,62 \mathrm{a}$ & $11,93 \mathrm{a}$ \\
Consorciado & $22,08 \mathrm{~b}$ & $1,45 \mathrm{~b}$ & $9,06 \mathrm{~b}$ \\
CV\% & 45,45 & 61,94 & 21,65 \\
DMS & 10,92 & 1,00 & 1,45 \\
\hline
\end{tabular}

${ }^{1}$ Médias seguidas pela mesma letra, nas colunas para cada cultura, não diferem estatisticamente entre si pelo teste de Tukey ao nível de 5\% de probabilidade. MF: Massa fresca; MS: Massa seca; CR: comprimento da raiz; Cv: coeficiente de variação; DMS: Diferença mínima significativa.

Baseando-se no 'princípio da exclusão competitiva', descrito por Vandermeer (1990) citado por Oliveira et al. (2005), que diz que se duas espécies têm necessidades distintas, competem entre si fracamente, sobrevivendo no mesmo ecossistema, porém, quando as necessidades são similares, competem entre si fortemente, com tendência de uma delas se extinguir em um dado período de tempo. Neste trabalho a cultura do almeirão foi fortemente afetada, com uma redução de 15,34 toneladas no sistema de monocultivo. Outro fator, seria quanto ao espaçamento utilizado, devido à semeadura mais adensada entre plantas, e o menor espaçamento entre linhas, o que afetou o seu desenvolvimento no sistema consorciado. Harder, Heredia Zarate e Vieira (2005), utilizou espaçamentos de 0,10, 0,15, 0,20 m entre plantas por 0,10 entre linhas. Cecilio Filho et al. (2008), relata que indepentemente da época em que foi semeada, a rúcula não apresentou efeitos sobre o crescimento da chicória, atribuindo isso ao seu ciclo curto, pequena altura, área foliar e projeção de suas folhas sobre a chicória, características estas que permitiram a chicória se desenvolver sem sofrer a interferência da rúcula. Em se tratando de eficiência no consorcio de plantas, Montezano e Peil (2006), afirmam que poderá ser considerado eficiente o consórcio quando o IEA for superior a 1,0, e considerado prejudicial quando for inferior a 1,0, quando for obtido qualquer valor maior que 1,0 indica uma vantagem do sistema de cultivo consorciado ao monocultivo, denominando esse resultado de sobreprodutividade. Para Fageria (1989) e Vandermeer (1990) citados por Oliveira et al. (2005), o sistema de cultivo consorciado só é considerado eficiente quando o indice for superior a 1,0, e desde que as culturas consorciadas atinjam um padrão comercial aceitável. Esse trabalho pode ser considerado eficiente em razão do Índice de Equivalência de Área (IEA), que alçançou o valor de 1,17 (Tabela 2). Isso significa que seria necessário um acréscimo de $17 \%$ de área plantada 
(espaço fisico) para se obter, no sistema de monocultivo, uma produtividade equivalente às alcançadas no sistema de cultivo consorciado. Segundo Vieira (1984) citado por Montezano e Peil (2006), o uso eficiente da terra expresso pelo índice de equivalência de área (IEA), tem sido utlizado com frequência, na avaliação da eficiência do consórcio em relação aos monocultivos, permitindo avaliar a eficiência biológica dos sistemas consorciados.

Tabela 2: Produtividade das culturas da rúcula e almeirão em monocultivo e consorciado.

\begin{tabular}{ccc}
\hline & \multicolumn{2}{c}{ Produtividade $\left(\mathbf{t ~ h a}^{-\mathbf{1}}\right)$} \\
\cline { 2 - 3 } Modo de Cultivo & Rúcula & Almeirão \\
\hline Monocultivo & $29,16 \mathrm{a}^{1}$ & $25,37 \mathrm{a}$ \\
Consorciado & $22,66 \mathrm{a}$ & $10,03 \mathrm{~b}$ \\
CV\% & 19,63 & 7,33 \\
DMS & 11,53 & 7,32 \\
Índice de Equivalência de Área (IEA) & 1,17 \\
\hline mesma letra, nas colunas para cada cultura, não diferem estatisticamente entre si pelo teste de
\end{tabular}

${ }^{1}$ Médias seguidas pela mesma letra, nas
Tukey ao nível de $5 \%$ de probabilidade.

\section{CONCLUSÃO}

De acordo com os resultados obtidos e a metodologia utilizada neste trabalho, para a cultura da rúcula, o melhor resultado foi obtido no monocultivo com uma produção de massa fresca de 42,77 g/planta, superior em 9,59 g/planta ao sistema de cultivo consorciado. Na cultura do almeirão, o melhor resultado foi obtido no monocultivo com uma produção de massa fresca de 52,881 g/planta, superior em $30,73 \mathrm{~g} /$ planta ao sistema de cultivo consorciado. Quanto ao sistema de cultivo consorciado, a rúcula não apresentou interferência significativa quando cultivada com o almeirão, e o almeirão por sua vez apresentou interferência significativa, obtendo redução em todos os itens avaliados. O índice de equivalência da área (IEA) atingiu 1,17 atestando maior eficiência do uso da área. Dessa forma pode-se concluir que o cultivo consorciado da rúcula com o almeirão foi adequado do ponto de vista agronômico, pois a presença do almeirão não prejudicou a produção da rúcula, apresentando uma possibilidade concreta de geração de renda extra para o agricultor em uma mesma área física.

\section{REFERÊNCIAS}

ASSISTAT. Software Estatístico. Disponível em: http://assistat.com . Acesso em: 20 out 2012.

CAETANO, L.C.S.; FERREIRA, J.M.; ARAÚJO, M.L. de. Produtividade de cenoura e alface em sistema de consorciação. Horticultura Brasileira, Brasília, v.17, n.2, p.143-146, 1999.

CECÍLIO FILHO, A, B.et al. Viabilidade produtiva e econômica do consórcio entre chicória e rúcula em função da época de plantio. Horticultura Brasileira, Brasília, v. 26, p. 316-320. 2008.

CECÍLIO FILHO, A. B. Cultivo consorciado de hortaliças: desenvolvimento de uma linha de pesquisa. Jaboticabal: UNESP/FCAV, 2005, 135 p.

CECÍLIO FILHO, A. B.; MAY, A. Produtividade das culturas de alface e rabanete em função da época de estabelecimento do consórcio, em relação a seus monocultivos. Horticultura Brasileira, Brasília, v. 20, p. 501-504. 2002. 
HARDER, W. C.; HEREDIA ZARATE, N. A.; VIEIRA, M. do C. Produção e rentabilidade de rúcula "cultivada" e de almeirão "amarelo" em cultivo solteiro e consorciado. Revista Ciência e Agrotecnologia, Lavras, v. 29, n. 4, p. 775-785, jul./ago. 2005.

HEREDIA ZÁRATE, N.A.et al. Produção e renda bruta de cebolinha e de salsa em cultivo solteiro e consorciado. Horticultura Brasileira, Brasília, v. 21, n. 3, p. 574-577, 2003.

MONTEZANO, E. M.; PEIL, R. M. N. Sistemas de consórcio na produção de Hortaliças. Revista Brasileira Agrociência, Pelotas, v. 12, n. 2, p. 129-132, 2006.

NEGREIROS, M. Z.et al. Cultivares de alface em sistemas solteiro e consorciado com cenoura em Mossoró. Horticultura Brasileira, Brasília, v. 20, n. 2, p. 162-166, 2002.

OLIVEIRA, F. L.et al. Desempenho do consórcio entre repolho e rabanete com pré-cultivo decrotalária, sob manejo orgânico. Horticultura Brasileira, Brasília, v. 23, n. 2, p. 184-188, 2005.

OLIVEIRA, E. Q. L.et al. Produtividade de alface e rúcula, em sistema consorciado, sob adubação orgânica e mineral. Horticultura Brasileira, v. 28, n.1, p. 36-40, 2010.

PORTO, V. C. N. Bicultivo de alface, cenoura e rúcula consorciadas com cenoura em faixas. 97 p, 2008.Tese (Doutorado em Fitotecnia) - Universidade Federal Rural do Semiárido (UFERSA), Mossoró.

TEIXEIRA FILHO, I. R.; MOTA. J. H.; SILVA, A. G. Consórcio de Hortaliças. Semina: Ciências Agrárias, Londrina, v. 26, n.4, p. 507-514, 2005.

VIEIRA, C. Índice de equivalência de área. Informe Agropecuário, Belo Horizonte, v. 10, n. 118, p. 1213, 1984. 